\title{
Effects of gastric bypass surgery on glucose absorption and metabolism during a mixed meal in glucose-tolerant individuals
}

\author{
Siv H. Jacobsen - Kirstine N. Bojsen-Møller • Carsten Dirksen • Nils B. Jørgensen • \\ Trine R. Clausen • Birgitte S. Wulff • Viggo B. Kristiansen • Dorte Worm • \\ Dorte L. Hansen • Jens J. Holst • Gerrit van Hall • Sten Madsbad
}

Received: 4 June 2013 / Accepted: 3 July 2013 / Published online: 27 July 2013

(C) Springer-Verlag Berlin Heidelberg 2013

\begin{abstract}
Aims/hypothesis Roux-en-Y gastric bypass surgery (RYGB) improves glucose tolerance in patients with type 2 diabetes, but also changes the glucose profile in response to a meal in glucose-tolerant individuals. We hypothesised that the driving force for the changed postprandial glucose profiles after RYGB is rapid entry of glucose into the systemic circulation due to modified gastrointestinal anatomy, causing hypersecretion of insulin and other hormones influencing glucose disappearance and endogenous glucose production.

Methods We determined glucose absorption and metabolism and the rate of lipolysis before and 3 months after RYGB in
\end{abstract}

\footnotetext{
S. H. Jacobsen $(\varangle) \cdot K$. N. Bojsen-Møller • C. Dirksen · N. B. Jørgensen • D. Worm • D. L. Hansen - S. Madsbad Department of Endocrinology 541, Hvidovre Hospital, Kettegaard Allé 30, 2650 Hvidovre, Denmark e-mail: siv.hesse@hotmail.com
}

S. H. Jacobsen • K. N. Bojsen-Møller • C. Dirksen •

N. B. Jørgensen · J. J. Holst

Novo Nordisk Foundation Centre for Basic Metabolic Research, the Panum Institute, University of Copenhagen, Copenhagen, Denmark

N. B. Jørgensen · J. J. Holst • G. van Hall

Department of Biomedical Sciences, Faculty of Health Sciences, University of Copenhagen, Copenhagen, Denmark

T. R. Clausen $\cdot$ B. S. Wulff

Diabetes and Obesity Biology, Novo Nordisk, Måløv, Denmark

\section{B. Kristiansen}

Department of Surgical Gastroenterology, Hvidovre Hospital, Hvidovre, Denmark

G. van Hall

Clinical Metabolomics Core Facility,

Rigshospitalet, Copenhagen, Denmark obese glucose-tolerant individuals using the double-tracer technique during a mixed meal.

Results After RYGB, the postprandial plasma glucose profile changed, with a higher peak glucose concentration followed by a faster return to lower than basal levels. These changes were brought about by changes in glucose kinetics: (1) a more rapid appearance of ingested glucose in the systemic circulation, and a concomitant increase in insulin and glucagon-like peptide-1 secretion; (2) postprandial glucose disappearance was maintained at a high rate for a longer time after RYGB. Endogenous glucose production was similar before and after surgery. Postoperative glucagon secretion increased and showed a biphasic response after RYGB. Adipose tissue basal rate of lipolysis was higher after RYGB.

Conclusions/interpretation A rapid rate of absorption of ingested glucose into the systemic circulation, followed by increased insulin secretion and glucose disappearance appears to drive the changes in the glucose profile observed after RYGB, while endogenous glucose production remains unchanged.

Trial registration ClinicalTrials.gov NCT01559792.

Funding The study was part of the UNIK program: Food, Fitness \& Pharma for Health and Disease (see www. foodfitnesspharma.ku.dk). Funding was received from the Novo Nordisk foundation and the Strategic Research Counsel for the Capital Area and Danish Research Agency. The primary investigator received a $\mathrm{PhD}$ scholarship from the University of Copenhagen, which was one-third funded by Novo Nordisk.

Keywords Endogenous glucose production - Gastric bypass - Gastrointestinal hormone response - Glucagon-like peptide-1 · Glucose kinetics · Insulin sensitivity · Obesity · Postprandial absorption $\cdot$ Rate of appearance $\cdot$ Rate of disappearance 


$\begin{array}{ll}\text { Abbreviations } \\ \text { EGP } & \text { Endogenous glucose production } \\ \text { FFM } & \text { Fat free mass } \\ \text { GIP } & \text { Glucose-dependent insulinotropic polypeptide } \\ \text { GLP-1 } & \text { Glucagon-like peptide-1 } \\ \text { IAUC } & \text { Incremental AUC } \\ \mathrm{R}_{\mathrm{a}} & \text { Rate of appearance } \\ \mathrm{R}_{\mathrm{d}} & \text { Rate of disappearance } \\ \text { RYGB } & \text { Roux-en-Y gastric bypass surgery }\end{array}$

\section{Introduction}

Roux-en-Y gastric bypass surgery (RYGB) induces remission of type 2 diabetes mellitus [1] often a few days after surgery [2], and, in glucose-tolerant individuals, the postprandial glucose profile changes, with a higher peak concentration and a faster return to basal glucose level [3-5]. We hypothesised that the driving force for the changed glucose profile after RYGB is the high rate of glucose entry into the systemic circulation due to modified gastrointestinal anatomy.

The aim of this study was to determine glucose absorption and metabolism, as well as lipolysis and hormonal changes, after a mixed meal before and 3 months after RYGB in glucose-tolerant individuals.

\section{Methods}

Participants The study included ten obese glucose-tolerant patients undergoing laparoscopic RYGB at Hvidovre Hospital (Copenhagen, Denmark). All patients successfully accomplished a preoperative diet-induced $8 \%$ loss in body weight. One patient experienced postoperative complications and was excluded from the study.

Written, informed consent was obtained from all patients, and the study was approved by the Municipal Ethics Committee of Copenhagen in accordance with the Helsinki-II Declaration and was registered at www.clinicaltrials.gov (ID NCT01559792).

Study design Patients were studied within 2 weeks before and 3 months after RYGB. Dual energy x-ray absorptiometry was used to assess fat-free mass (FFM). On each study day, patients arrived after an overnight fast (10-12 h), and intravenous cannulas were inserted into both arms. A primed continuous infusion of $\left[6,6-\mathrm{D}_{2}\right]$ glucose $(17.6 \mu \mathrm{mol} / \mathrm{kg}$ FFM, $0.4 \mu \mathrm{mol}[\mathrm{kg} \mathrm{FFM}]^{-1} \mathrm{~min}^{-1}$ ) and $\left[1,1,2,3,3-\mathrm{D}_{5}\right]$ glycerol $\left(1.5 \mu \mathrm{mol} / \mathrm{kg} \mathrm{FFM}, 0.1 \mu \mathrm{mol}[\mathrm{kg} \mathrm{FFM}]^{-1} \mathrm{~min}^{-1}\right)$ (Cambridge Isotope Laboratories, Andover, MA, USA) was initiated. After $2 \mathrm{~h}$ of tracer infusion, the steady-state glucose and glycerol concentration and enrichment were determined, and a mixed meal
(200 ml, 1,650 kJ (394 kcal): carbohydrate $50 \%$, protein $15 \%$, fat $35 \%$, consisting of glucose $\left(48.4 \mathrm{~g}+1.6 \mathrm{~g}\left[\mathrm{U}_{-}^{13} \mathrm{C}\right]\right.$ glucose $)$, rapeseed oil $[14.1 \mathrm{~g}]$ and casein protein $[15.2 \mathrm{~g}])$ was provided. The meal was consumed slowly over $30 \mathrm{~min}$.

Blood was sampled before the start of the continuous tracer infusion and at $-30,-15,0,15,30,45,60,90,120$, 180 and 240 min relative to meal intake. Urine was sampled throughout the study for assessment of traces of glucose in order to estimate glucose excretion.

Blood samples and tracers were analysed and calculated as described previously $[4,6,7]$.

Incremental AUC (IAUC) was calculated using the trapezoidal method with subtraction of baseline concentrations. Fasting concentrations were measured before infusion of tracers and basal concentrations before meal ingestion. Insulin resistance was assessed applying HOMA-IR and the hepatic insulin resistance index, multiplying fasting insulin concentrations by fasting endogenous glucose production (EGP). Oral glucose recovery, i.e. percentage of oral glucose that appears in the systemic circulation, was calculated from IAUC of the oral glucose rate of appearance $\left(R_{a}\right)$ curve and expressed as a percentage of the amount of ingested glucose in the meal.

Data are expressed as mean \pm SEM. The Wilcoxon signed rank test was used to compare data before and after RYGB. $p$ values $<0.05$ were considered significant.

\section{Results}

Patient characteristics and baseline concentrations before and after RYGB Patients (three men, nine women) with a preoperative BMI of $39.2 \pm 1.8 \mathrm{~kg} / \mathrm{m}^{2}$ were examined $-4 \pm 2$ days before the operation. Three months ( $92 \pm$ 4 days) after surgery, patients had a significant weight loss of $18.5 \pm 0.6 \mathrm{~kg}$, and BMI had decreased by $6.3 \pm 0.3 \mathrm{~kg} / \mathrm{m}^{2}$. FFM was reduced by $-6.6 \pm 1.0 \mathrm{~kg}$, and fat mass by $-10.5 \pm 1.1 \mathrm{~kg}$, leading to a reduction in body fat percentage $(-3.5 \pm 1.1 \%)$.

Fasting glucose, insulin and C-peptide concentrations were lower after RYGB $(5.2 \pm 0.2$ vs $4.9 \pm 0.1 \mathrm{mmol} / \mathrm{l}, p=0.008$; $125 \pm 16$ vs $66 \pm 7 \mathrm{pmol} / \mathrm{l}, p=0.008 ; 920 \pm 118$ vs $684 \pm$ $64 \mathrm{pmol} / \mathrm{l}, p=0.027$, respectively), while glucagon and glucose-dependent insulinotropic polypeptide (GIP) levels were unchanged (7.2 \pm 0.6 vs $7.2 \pm 0.8 \mathrm{pmol} / \mathrm{l}, p=1.0 ; 13 \pm 2$ vs $12 \pm 2 \mathrm{pmol} / \mathrm{l}, \quad p=0.383), \quad$ and glucagon-like peptide-1 (GLP-1) increased ( $9 \pm 1 \mathrm{vs} 11 \pm 1 \mathrm{pmol} / \mathrm{l}, p=0.031)$. HOMAIR and hepatic insulin resistance index decreased $(4.3 \pm 0.6$ vs $2.1 \pm 0.2, p=0.008 ; 108 \pm 15$ vs $57 \pm 5[\mathrm{mmol} / \mathrm{min}] \times[\mathrm{pmol} / 1]$, $p=0.012$ ), but basal EGP was unchanged after RYGB $(0.99 \pm 0.04$ vs $1.06 \pm 0.04 \mathrm{mmol} / \mathrm{min}, p=0.203)$. Expressed per kg FFM, EGP increased after RYGB, explained by a decrease in FFM. 
Glucose kinetics The postprandial peak glucose concentration was substantially higher after RYGB $(p=0.004)$, but with no change in time to peak $(p=0.313)$ (Fig. 1a). The subsequent decrease in glucose concentration was much faster after RYGB and reached basal levels $2 \mathrm{~h}$ after meal ingestion (basal to $2 \mathrm{~h}$ value, $p=0.250$ ) compared with at $3 \mathrm{~h}$ before RYGB (basal to $3 \mathrm{~h}$ value, $p=0.074$ ). Furthermore, after RYGB, $3 \mathrm{~h}$ postprandial glucose concentrations reached a nadir that was lower than the preprandial basal concentrations $(p=0.008)$ and then slowly increased thereafter.

Postprandial EGP suppression during the first $3 \mathrm{~h}$ was similar before and after RYGB ( $66 \pm 3$ vs $63 \pm 2 \%, p=0.3$, from basal to nadir) (Fig. 1d). After 3 h, EGP increased again
Fig. 1 Plasma glucose concentration (a), rate of total glucose appearance in (b) and disappearance from (c) the systemic circulation, endogenous (d) and oral (e) rates of glucose appearance in the systemic circulation, plasma glycerol concentrations (f), and rate of glycerol appearance per $\mathrm{kg}$ fat mass (g) in the systemic circulation before (white squares) and $\sim 3$ months after (black squares) RYGB. First part of the curve illustrates the basal level and the second part the postprandial response. The dotted lines mark the mean preoperative basal levels
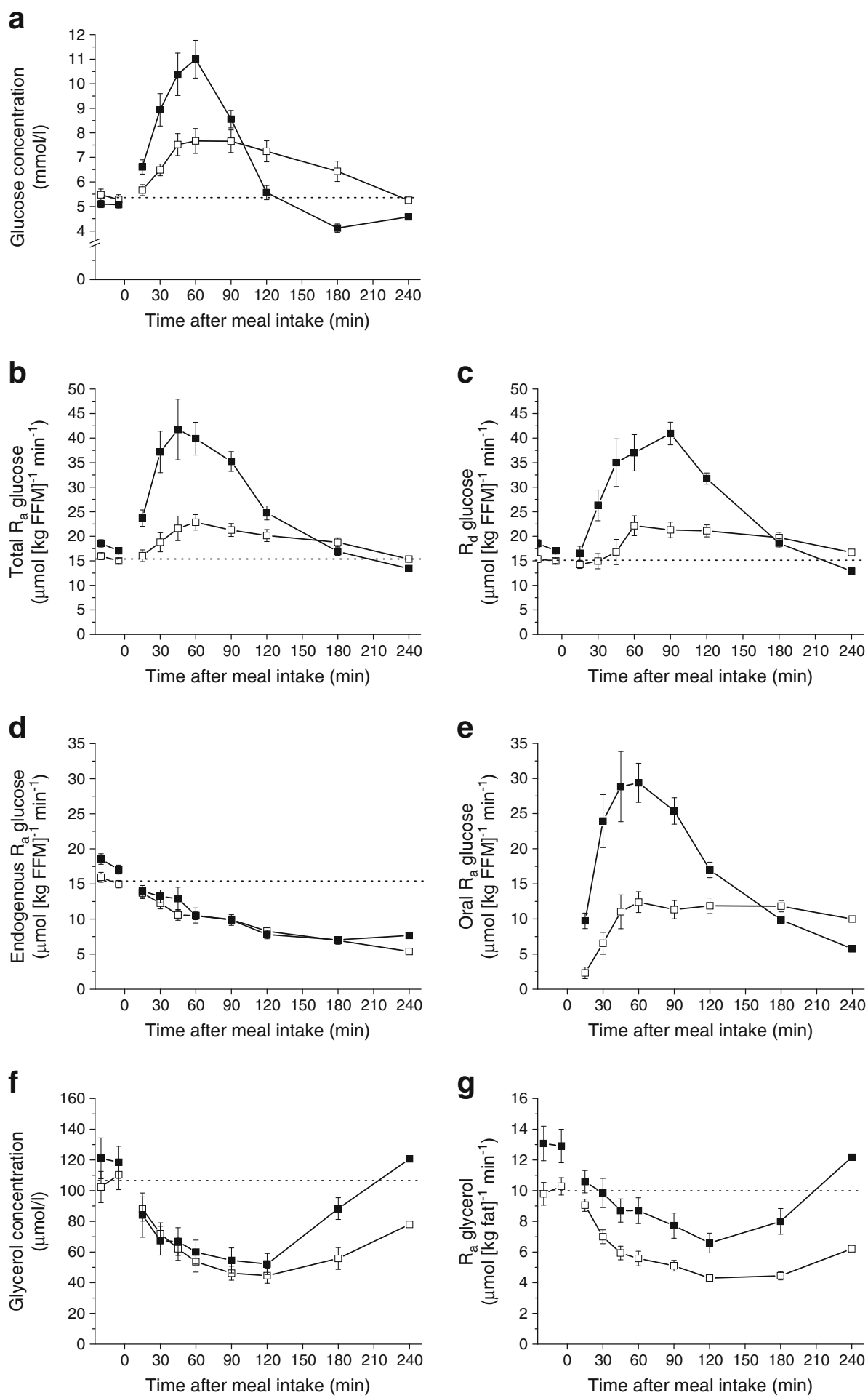
after surgery, and, at $4 \mathrm{~h}$, the rate was higher than before RYGB $(p=0.004)$.

Oral glucose $\mathrm{R}_{\mathrm{a}}$ increased after RYGB $(p=0.004$ for IAUC) (Fig. 1e), and oral glucose recovery changed from $54 \pm 4 \%$ to $80 \pm 2 \% \quad(p=0.004)$, equivalent to absorption of 27 and $40 \mathrm{~g}$ of the ingested glucose load within the $4 \mathrm{~h}$ study period before and after RYGB, respectively.

Postprandial glucose rate of disappearance $\left(R_{d}\right)$ also increased after RYGB ( $p=0.004$ for IAUC). Both before and after surgery, $R_{d}$ increased later and was elevated for longer than $\mathrm{R}_{\mathrm{a}}$, but postoperatively this time lag was more pronounced, resulting in larger differences between $R_{a}$ and $R_{d}$ (Fig. 1b, c), which explains the higher peak and lower nadir plasma glucose concentrations after RYGB.
Glycerol kinetics Glycerol $\mathrm{R}_{\mathrm{a}}$ per fat mass (Fig. 1g), measured at basal 15 min before meal ingestion increased after RYGB $(p=0.02)$, although the postprandial suppression of $\mathrm{R}_{\mathrm{a}}$ glycerol was similar before and after RYGB $(p=0.652$, from basal to nadir).

Hormones IAUC for insulin and C-peptide were higher after RYGB ( $p=0.004$ for both) (Fig. 2a, b).

Postoperatively, glucagon levels were significantly elevated postprandially ( $p=0.027$ for IAUC) and the curve displayed a biphasic profile with a second peak $3 \mathrm{~h}$ after meal ingestion (Fig. 2c). The secretion of GLP-1 was elevated fourfold ( $p=0.004$ for IAUC) (Fig. 2d), and the GIP profile changed with a shift to the left, with a higher peak value $(p=0.039)$ occurring earlier after the operation ( $81 \pm 10$ vs $48 \pm 3$ min, $p=0.02$ ) (Fig. $2 \mathrm{e}$ ).
Fig. 2 Plasma hormone concentrations after ingestion of a mixed meal before (white squares) and $\sim 3$ months after (black squares) RYGB. First part of the curve illustrates the basal level and the second part the postprandial response. The dotted lines mark the mean preoperative basal levels
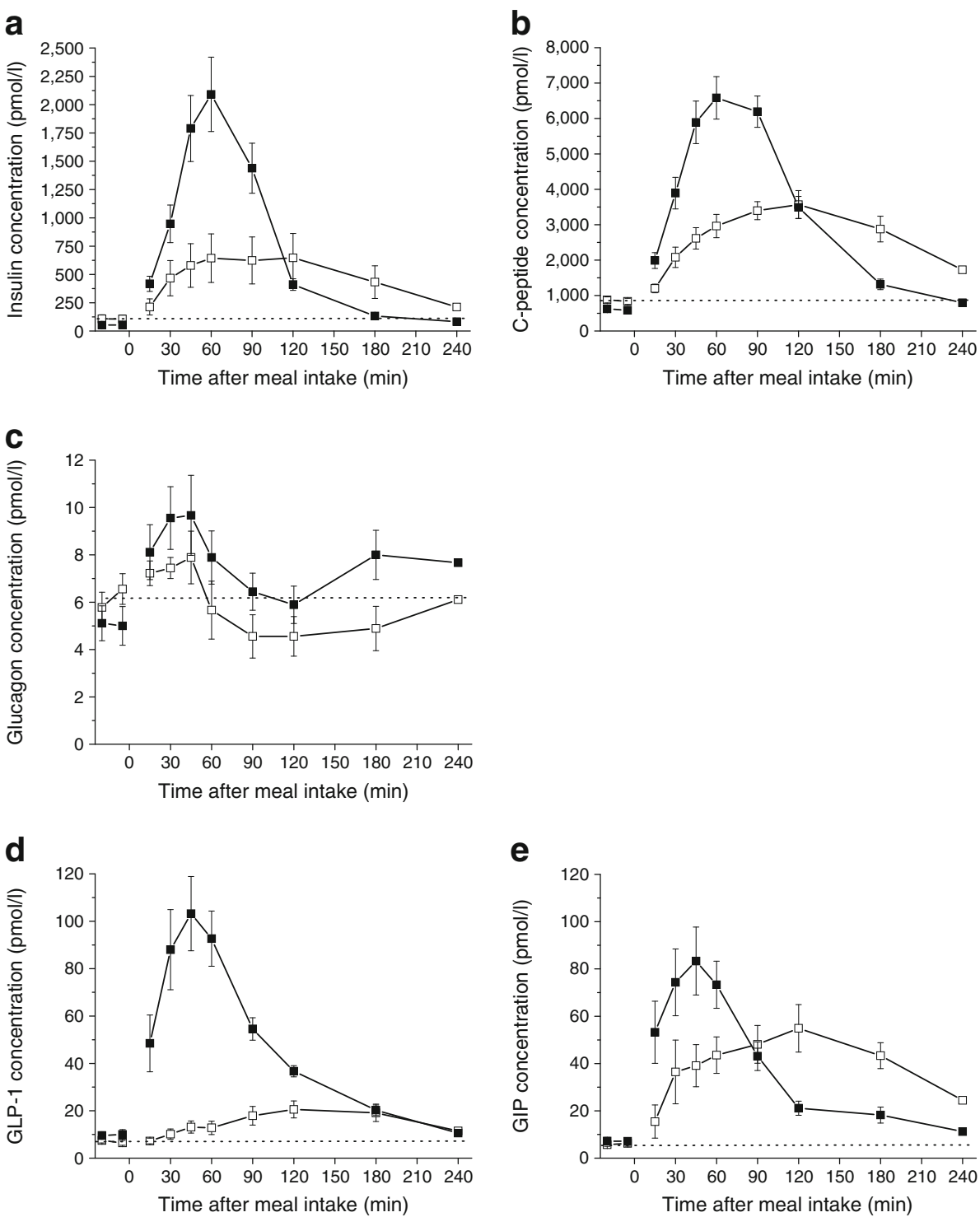


\section{Discussion}

Our key finding was a rapid postprandial appearance of a larger amount of orally ingested glucose in the systemic circulation after RYGB. Postprandial glucose disappearance was also increased, but lagged behind glucose appearance, causing an increase in postprandial plasma glucose concentration. This time lag was much more pronounced after RYGB. The later decrease in plasma glucose was partly due to decreasing oral glucose $\mathrm{R}_{\mathrm{a}}$, but more importantly also to enhanced and prolonged glucose disappearance in response to hypersecretion of insulin, possibly due to exaggerated GLP-1 release [8, 9]. As a consequence, the glucose concentration had already reached the basal level $2 \mathrm{~h}$ postprandially, followed by a brief period of hypoglycaemia. The hepatic insulin resistance index decreased after RYGB, indicating an increase in hepatic insulin sensitivity. However, basal and postprandial EGP was unaffected by RYGB despite threefold higher postprandial insulin levels. Postoperative glucagon secretion showed a biphasic response, with an initial increase and a second peak during the transient hypoglycaemic period. This may have counteracted the insulin action on EGP and also illustrates the importance of an intact alpha cell-liver interaction to prevent postprandial hypoglycaemia after RYGB. Our data are somewhat different from the recent study of Bradley et al [10], who reported faster and almost complete initial suppression of EGP after RYGB with a rapid return to baseline levels and a nearcomplete meal glucose appearance in the circulation within the first postprandial hour. However, the meal composition was different, and no changes in glucagon concentrations after surgery were found.

Fast delivery of ingested glucose into the distal part of the small intestine may provoke the increased GLP-1 response, which in combination with the exaggerated glucose concentration probably explains the increase in insulin secretion [9]. This seems to be one of the keystones in the mechanism of improved glucose tolerance after RYGB in patients with type 2 diabetes [8].

Basal glycerol $\mathrm{R}_{\mathrm{a}}$ into the circulation, a measure of endogenous lipolytic rate, was increased after RYGB in agreement with the lower fasting insulin levels. However, initial suppression of lipolysis in the postprandial phase was unaffected by RYGB in spite of much higher systemic insulin concentrations. Thus, after RYGB, postprandial fat tissue insulin sensitivity seems reduced.

In conclusion, meal ingestion after RYGB elicited higher peak plasma glucose concentrations followed by an earlier and more pronounced decrease to levels even below basal. The driving forces were higher $\mathrm{R}_{\mathrm{a}}$ of ingested glucose and delayed but enhanced and prolonged glucose disappearance due to insulin and GLP-1 hypersecretion. EGP was mainly unaffected by RYGB. Basal lipolytic rates increased after RYGB.
Acknowledgements This work would not have been possible without the professional help of Alis Sloth Andersen and Dorthe Baunbjerg Nielsen at the Department of Endocrinology, Hvidovre Hospital, Denmark and Lene Brus Albæk at the Department of Biomedical Sciences, Faculty of Health Sciences, University of Copenhagen, Denmark, as well as Nina Pluszek and Lene Foged at the Clinical Metabolomics Core Facility, Rigshospitalet, Copenhagen, Denmark.

Funding This work was part of the UNIK program: Food, Fitness \& Pharma for Health and Disease (see www.foodfitnesspharma.ku.dk). Funding was received from the Novo Nordisk foundation and the Strategic Research Counsel for the Capital Area and Danish Research Agency. The primary investigator received a $\mathrm{PhD}$ scholarship from the University of Copenhagen, which was one-third funded by Novo Nordisk

Duality of interest The authors declare that there is no duality of interest associated with this manuscript.

Contribution statement SHJ, KNB-M, SM, JJH and GvH designed the study; SHJ performed the research; SHJ, KNB-M, CD, NBJ, DLH, DW, VBK, BSW, TRC, JJH, GvH and SM contributed to data analysis and/or interpreted the results; SHJ wrote the manuscript; SHJ, KNB-M, CD, NBJ, DLH, DW, VBK, BSW, TRC, JJH, GvH and SM revised and approved the final version of the manuscript.

\section{References}

1. Sjostrom L, Lindroos AK, Peltonen M et al (2004) Lifestyle, diabetes, and cardiovascular risk factors 10 years after bariatric surgery. N Engl J Med 351:2683-2693

2. Pories WJ, Swanson MS, MacDonald KG et al (1995) Who would have thought it? An operation proves to be the most effective therapy for adult-onset diabetes mellitus. Ann Surg 222:339-350

3. Falken Y, Hellstrom PM, Holst JJ, Naslund E (2011) Changes in glucose homeostasis after Roux-en-Y gastric bypass surgery for obesity at day three, two months, and one year after surgery: role of gut peptides. J Clin Endocrinol Metab 96:2227-2235

4. Jacobsen SH, Olesen SC, Dirksen C et al (2012) Changes in gastrointestinal hormone responses, insulin sensitivity, and betacell function within 2 weeks after gastric bypass in non-diabetic subjects. Obes Surg 22:1084-1096

5. Jorgensen NB, Jacobsen SH, Dirksen C et al (2012) Acute and long-term effects of Roux-en-Y gastric bypass on glucose metabolism in subjects with type 2 diabetes and normal glucose tolerance. Am J Physiol Endocrinol Metab 303:E122-E131

6. Gastaldelli A, Casolaro A, Pettiti M et al (2007) Effect of pioglitazone on the metabolic and hormonal response to a mixed meal in type II diabetes. Clin Pharmacol Ther 81:205-212

7. Wolsk E, Mygind H, Grondahl TS, Pedersen BK, van Hall G (2010) IL-6 selectively stimulates fat metabolism in human skeletal muscle. Am J Physiol Endocrinol Metab 299:E832-E840

8. Dirksen C, Jorgensen NB, Bojsen-Moller KN et al (2012) Mechanisms of improved glycaemic control after Roux-en-Y gastric bypass. Diabetologia 55:1890-1901

9. Salehi M, Prigeon RL, D'Alessio DA (2011) Gastric bypass surgery enhances glucagon-like peptide 1-stimulated postprandial insulin secretion in humans. Diabetes 60:2308-2314

10. Bradley D, Conte C, Mittendorfer B et al (2012) Gastric bypass and banding equally improve insulin sensitivity and beta cell function. J Clin Invest 122:4667-4674 